\title{
Secondary Endpoint
}

National Cancer Institute

\section{Source}

National Cancer Institute. Secondary Endpoint. NCI Thesaurus. Code C139173.

An auxiliary endpoint associated with the study or trial. 